\title{
Experimentelle Untersuchungen zur wässrigen Kreuzstromextraktion von Krappwurzelfarbstoff
}

\author{
Jens-Uwe Gerking, Torsten Herfort, Eckhard Meyer, Josef Schmadl
}

\section{Einleitung}

Pflanzenfarbstoffe kommen derzeit nur in Spezialanwendungen zum Einsatz, da synthetische Farbstoffe wesentlich kostengünstiger erhältlich sind. Das Interesse an Naturfarbstoffen steigt jedoch zusehends mit wachsendem Umwelt- und Gesundheitsbewusstsein der Menschen, z. B. in der Lebensmittel- und Textilindustrie. Herkömmliche Färbetechniken sind für industrielle Anwendungen ungeeignet, da früher mit der ganzen Pflanzenmasse manuell gefärbt wurde, für den Einsatz mit heutigen Färbemaschinen aber nur konditionier- und dosierfähige, in der Regel volllösliche Farbstoffe in Frage kommen. Auch der in dieser Arbeit untersuchte, als „Färberröte“ seit jeher bekannte, rote Krappwurzelfarbstoff war im „Chemiezeitalter" nahezu in Vergessenheit geraten. Seit einigen Jahren ist er wieder vermehrt Gegenstand von Forschungsarbeiten sowohl in der Landwirtschaft als nachwachsender Rohstoff, wie auch in der Textilfärbung und in der verfahrenstechnischen Wertstoffabtrennung. Halbquantitative Vorversuche $[1,2]$ mit einer einfachen Apparatur hatten gezeigt, dass der Krappwurzelfarbstoff mit drei Extraktionsstufen im wässrigen Kreuzstrom mit guter Ausbeute extrahierbar war und bei Extraktionstemperaturen um $90{ }^{\circ} \mathrm{C}$ die besten Färbeergebnisse lieferte. In dieser Arbeit werden Einflüsse verschiedener verfahrenstechnischer Parameter des Extraktionsprozesses auf Farbstoffausbeute, Extraktionskinetik und Färbequalität quantitativ untersucht als Beitrag zur Entwicklung eines Industrieverfahrens zur Isolierung eines konditionierfähigen Färbeproduktes.

\section{Versuchstechnik}

Die verwendete Miniplant-Technikumsapparatur ist in Abbildung 1 vereinfacht dargestellt. Es handelt sich um einen mit Thermoöl beheizbaren Kreislauf mit Extraktvorlage, Pumpe und Extraktor (wahlweise $5 \mathrm{l}$ und $15 \mathrm{l}$ ) mit UV-Vis-Online-Analytik, für die ein Spektralphotometer vom Typ V560 der Firma Jasco mit einer 0,1 mm -Durchflussküvette eingesetzt wurde. Für die Extraktion wurde jeweils Extraktionsmittel vorgelegt und auf die gewünschte Extraktionstemperatur erwärmt. Nach Erreichen der Solltemperatur wurden zunächst der Messvorgang und unmittelbar darauf die Extraktion gestartet. Der Prozess wurde isotherm durchgefuihrt und anhand der UV-Vis-Absorption bei konstanter Wellenlänge oder - je nach Versuchsziel - anhand des im Intervall-Scan aufgenommenen Gesamtspektrums im Be- reich 200 - $600 \mathrm{~nm}$ kontrolliert. Versuchsende war das Erreichen der theoretischen Trennstufe entsprechend dem Sorptionsgleichgewicht mit einem Absorptionsmaximum bei konstanter Wellenlänge.

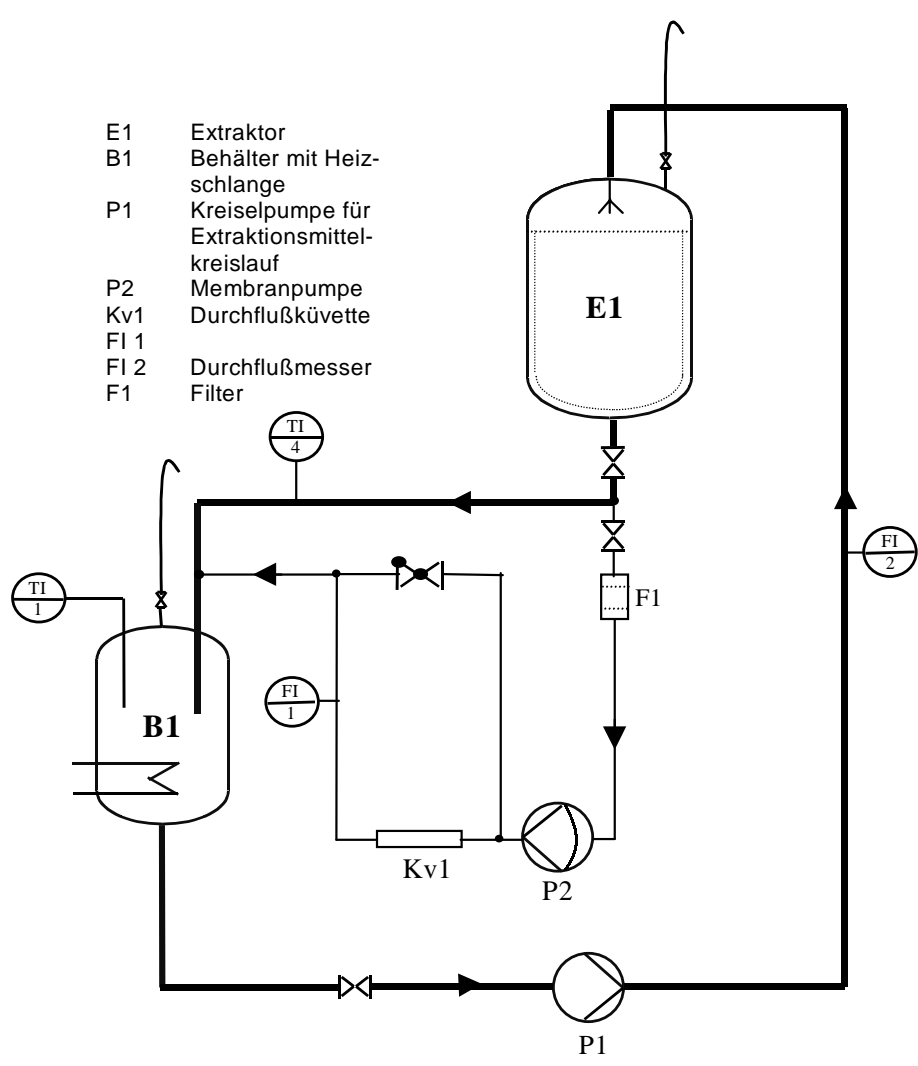

Abb. 1: Extraktionsapparatur mit on-line-Messschleife für die UV-Vis-Spektrophotometrie

Abbildung 2 zeigt die Spektren der wichtigsten, für Krapp bekannten Einzelfarbstoffe aus der Gruppe der Anthrachinon-Farbstoffe im Vergleich zum Spektrum eines wässrigen Krapp-Extraktes. Obwohl der Farbstoffanteil im Extrakt nur etwa 10 \% der gesamten Trockenmassse ausmacht, dominieren die Farbstoffe das UVVis-Spektrum, welches sich im Wesentlichen als eine Überlagerung der Einzelspektren von Alizarin, Ruberythrinsäure und Purpurin darstellt. Ein Absorptionsmaximum der Einzelspektren liegt jeweils bei $260 \mathrm{~nm}$. Insbesondere im Wellenlängenbereich zwischen 400 und $500 \mathrm{~nm}$ unterscheiden sich die Maxima der Einzelsubstanzen jedoch deutlich. Die Überlagerung bewirkt daher, dass im Spektrum eines wässrigen Krapp-Extraktes zwischen 400 und $500 \mathrm{~nm}$ ein relativ flacher, breiter Peak, bei $260 \mathrm{~nm}$ dagegen ein gut ausgeprägtes Maximum erscheint. Zum steilen Anstieg im Bereich unter $220 \mathrm{~nm}$ tragen vor allem nicht färbende Inhaltsstoffe im Krapp-Extrakt bei. 


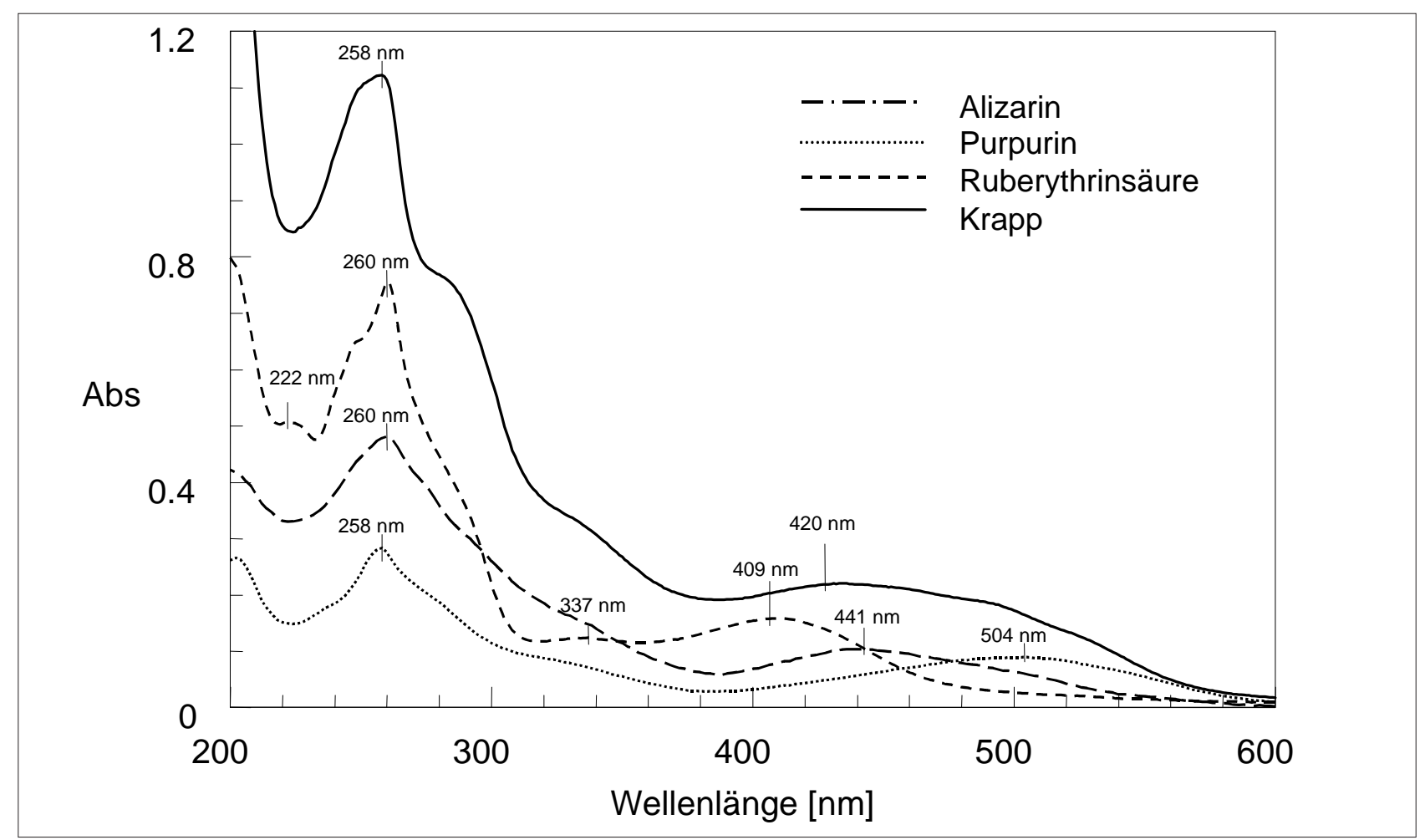

Abb. 2: UV-Vis-Spektren wässriger Lösungen der wichtigsten Krapp-Einzelfarbstoffe im Vergleich zu Krapp-Extrakt.

Abbildung 3 zeigt ein typisches, im Intervall-Scan-Verfahren aufgenommenes Spektrum. Während der Extrakt die Durchflussküvette durchströmt, wird vom Spektrometer in vorgegebenen Zeitabständen ein Spektrum im Bereich 200...600 nm aufgenommen. In der Auswertung wurde daraus der zeitliche Verlauf der Absorption (Extinktion) bei einer beliebigen Wellenlänge ermittelt. Außerdem konnten mit dieser Technik zeitliche Verschiebungen der Absorptionsmaxima im Verlaufe des Extraktionsprozesses, bedingt durch die komplexe Koextraktion verschiedener Farbstoffkomponenten, beobachtet werden.

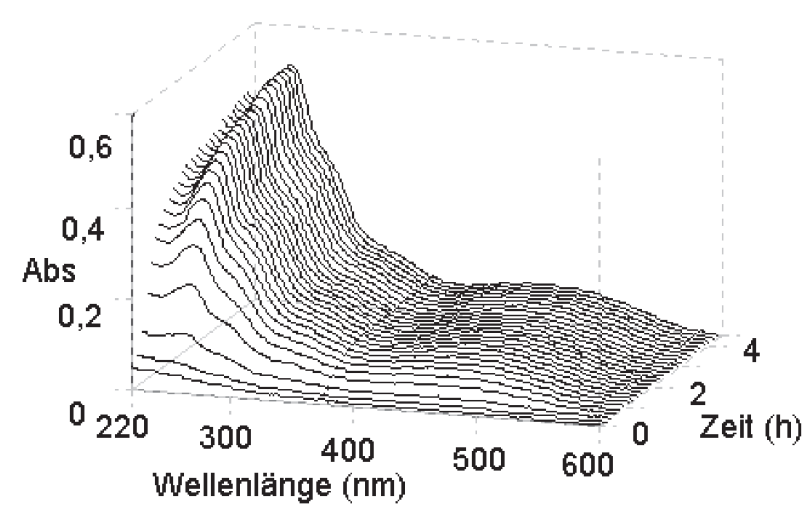

Abb. 3: Typische Intervall-Scan-Messung eines Krapp-Extraktionsversuches bei $T_{\text {extr }}=90^{\circ} \mathrm{C}$.

\section{Einfluss der Stufenzahl auf Extraktions- ausbeute und Extraktzusammensetzung}

Abbildung 4 zeigt das UV-Vis-Spektrum fuir drei aus einer Reihe von acht Extraktionsstufen bei $90{ }^{\circ} \mathrm{C}$, gemessen jeweils am Ende des Extraktionsprozesses. Das Maximum bei 420 nm deutet auf Ruberythrinsäure als dominieren- den Farbstoff in der ersten Stufe hin, was sich mit der besseren Löslichkeit im Vergleich zum reinen Alizarin erklären lässt. In den folgenden Stufen verschiebt sich das Maximum zu höheren Wellenlängen in einen Bereich, in dem bevorzugt das reine Alizarin, aber auch Pupurin absorbiert. Eine Erhöhung der Alizarin-Konzentration auf Kosten der Ruberythrinsäure wird daneben durch Abspal-

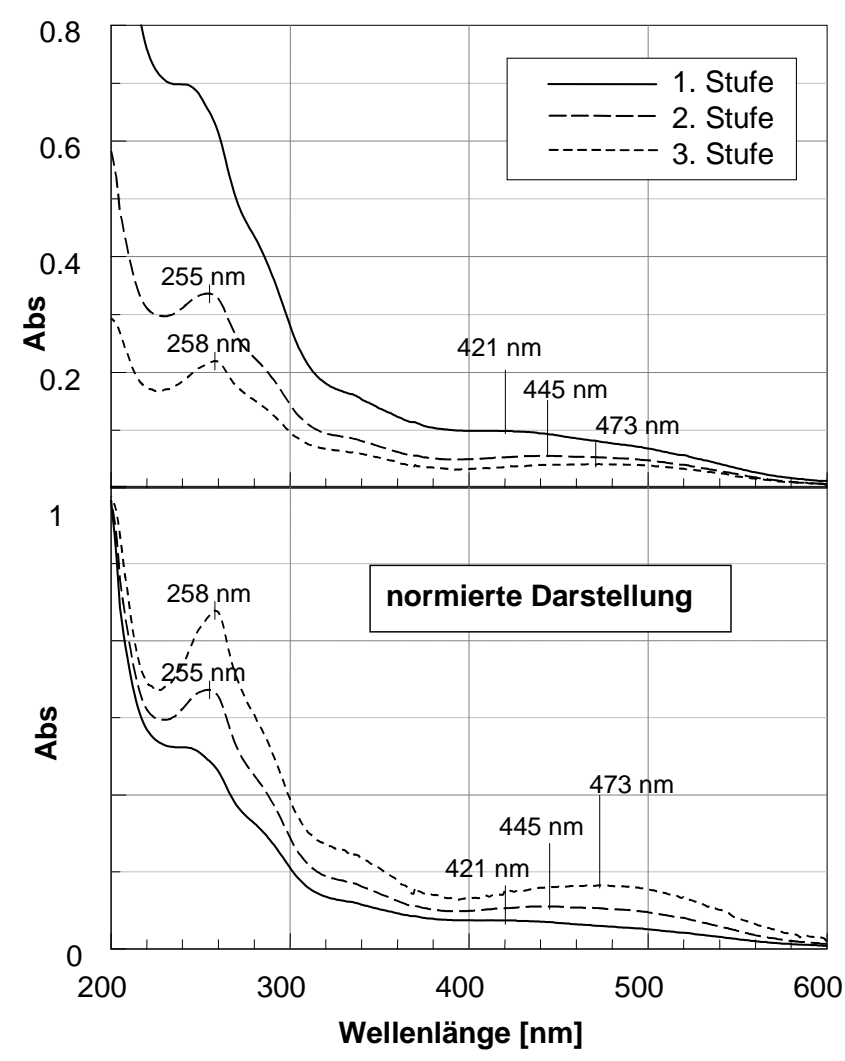

Abb. 4: UV-Vis-Spektren der Extrakte Nr. 1, 2 und 3 aus einer achtstufigen Kreuzstromextraktion bei $T_{\text {extr }}=90{ }^{\circ} \mathrm{C}$ als Absorption (oben) und normierte Absorption (unten) in Abhängigkeit von der Wellenlänge. 
tung des Zuckers von der Ruberythrinsäure, begünstigt durch die hohen Temperaturen und den im Verlauf der Extraktion absinkenden pH-Wert, hervorgerufen.

Auch bei niedrigen Wellenlängen erfährt das Spektrum von Stufe zu Stufe deutliche Veränderungen. Das Maximum bei $260 \mathrm{~nm}$ tritt mit zunehmender Stufenzahl stärker hervor, die Absorption unter $220 \mathrm{~nm}$, die in der ersten Stufe die Farbstoffabsorption bei $260 \mathrm{~nm}$ noch um ein Mehrfaches uibertraf, liegt ab der dritten Stufe nur noch geringfuigig darüber. Dieses Verhalten wird besonders in der normierten Darstellung deutlich. Daraus ergeben sich erste Hinweise auf in der ersten Extraktionsstufe bevorzugt koextrahierte und deshalb in erheblicher Konzentration vorhandene nicht färbende Begleitstoffe.

Abbildung 5 gibt die Extraktionsausbeute in Abhängigkeit von der Zahl der Extraktionsstufen bei $\mathrm{T}=90{ }^{\circ} \mathrm{C}$ an. Dabei wurden für acht Extraktionsstufen zunächst die jeweiligen Trockenmassen der Extrakte bestimmt und durch Extrapolation bis zu Stufe 15 weiter berechnet. Mit einem Bestimmtheitsmaß von 0,98 ergibt sich bei Stufe 15 eine extrahierte Trockenmasse von 0,26 g, das sind 0,2\% des Wertes der ersten Stufe. Mit dieser Näherung wurde für 15 Extraktionsstufen eine 100\%ige Extraktion angenommen. Davon ausgehend wurden die Ausbeuten der einzelnen Stufen berechnet und aufsummiert. Entsprechend Abbildung 5 werden nach drei Stufen $90 \%$ und nach acht Stufen $98 \%$ der Gesamtausbeute erzielt. Nicht beruicksichtigt ist dabei, welchen Anteil die Farbstoffe an der extrahierten Trockenmasse haben. Für die Ermittlung der Farbstoffausbeute wurde die Absorption bei $420 \mathrm{~nm}$ herangezogen und analog vorgegangen. Dabei ergab sich eine deutlich geringere Ausbeute. Nach acht Extraktionsstufen ist eine Ausbeute von $85 \%$ der auf 15 Stufen extrapolierten Gesamtfarbstoffmenge erreicht.

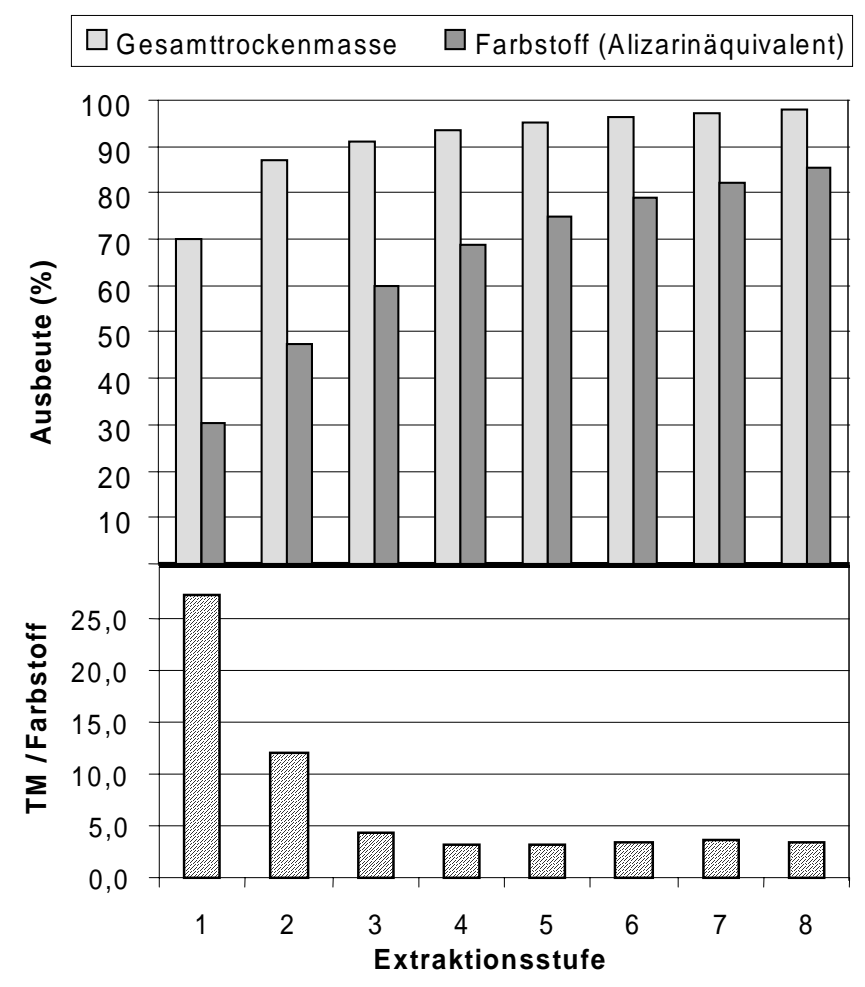

Abb. 5: Gesamtausbeute und Farbstoffausbeute (oben) sowie Verhältnis von extrahierter Trockenmasse zu Farbstoffmenge (unten) bei $T_{\text {extr }}=90^{\circ} \mathrm{C}$ in Abhängigkeit von der Extraktionsstufe
Die Farbstoffe in Krapp sind demnach deutlich schlechter wasserlöslich als andere, koextrahierte Inhaltsstoffe. Das Verhältnis der gesamten extrahierten Trockenmasse zum extrahierten Farbstoff beträgt entsprechend Abbildung 5 (unten) im Extrakt der ersten Stufe noch ca. 30, schon ab der dritten Stufe ist es auf unter fünf abgesunken und bleibt in den weiteren Stufen etwa konstant.

\section{Einfluss der Temperatur auf Extraktions- ausbeute und -kinetik}

In Abbildung 6 ist die Absorption bei $255 \mathrm{~nm}$ in Abhängigkeit von der Zeit jeweils für die 1. Extraktionsstufe bei verschiedenen Temperaturen zwischen 25 und $90{ }^{\circ} \mathrm{C}$ dargestellt.

Bis auf die - möglicherweise wegen einer Störung der UV-Vis-Messung - nur teilweise vergleichbare Extraktion bei $50{ }^{\circ} \mathrm{C}$ stellt sich bei allen untersuchten Temperaturen nach maximal $4 \mathrm{~h}$ ein konstanter Wert fuir die Absorption ein, der erwartungsgemäß mit steigender Temperatur zunimmt.

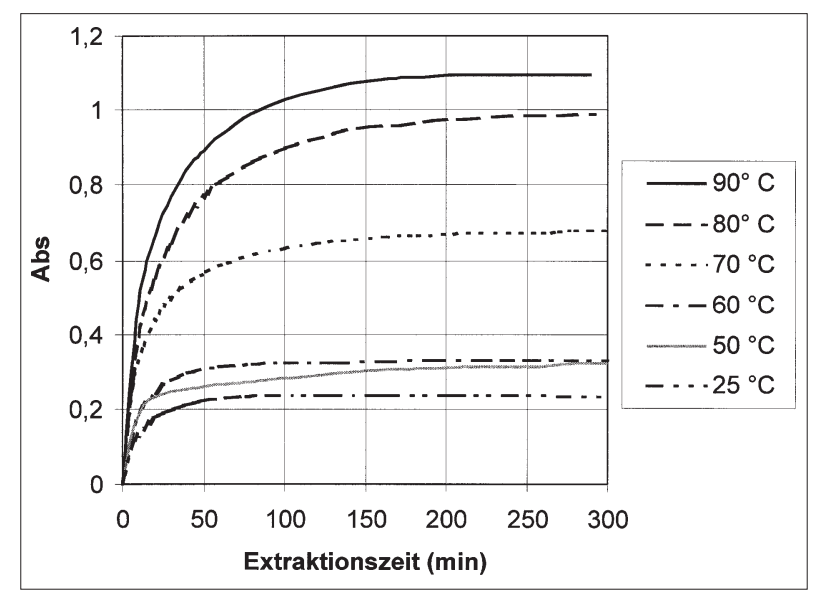

Abb. 6: Absorption bei konstanter Wellenlänge (255 nm) in Abhängigkeit von der Extraktionszeit für unterschiedliche Extraktionstemperaturen

Der Einfluss der Temperatur auf die gesamte extrahierte Trockenmassse ist gering, wie aus Abbildung 7 (oben) hervorgeht. Eine Temperaturerhöhung von 25 auf $90{ }^{\circ} \mathrm{C}$ bewirkt einen Anstieg der extrahierten, nach Trocknung gravimetrisch ermittelten Trockenmasse um lediglich $20 \%$. Der größte Teil der wasserlöslichen Inhaltsstoffe in Krapp ist demnach kaltwasserlöslich.

Betrachtet man die Farbstoffabsorption Abs $\infty$, in Abbildung 7 (unten), zeigt sich demgegenuiber ein deutlicher Temperatureinfluss, der zwischen 60 und $80^{\circ} \mathrm{C}$ zu einem sprunghaften Anstieg der Farbstoffausbeute fuihrt, so dass bei $90{ }^{\circ} \mathrm{C}$ eine um ca. Faktor 5 höhere Ausbeute als bei $25{ }^{\circ} \mathrm{C}$ erzielt wird. Da die UV-Vis-Absorption entsprechend Abbildung 2 aus einer Überlagerung der Einzelkomponenten hervorgeht, kann dieses Verhalten auf die unterschiedlichen Sorptionsisothermen der Einzelkomponenten zurückgefuihrt werden. Ein großer Teil der Inhaltsstoffe, u. a. Ruberythrinsäure, ist auch bei Raumtemperatur in Wasser gut löslich, während insbesondere Alizarin und Purpurin nur bei höheren Temperaturen in Lösung übergehen [3] und erst dann einen merklichen Einfluss auf das Gesamtspektrum ausüben. 


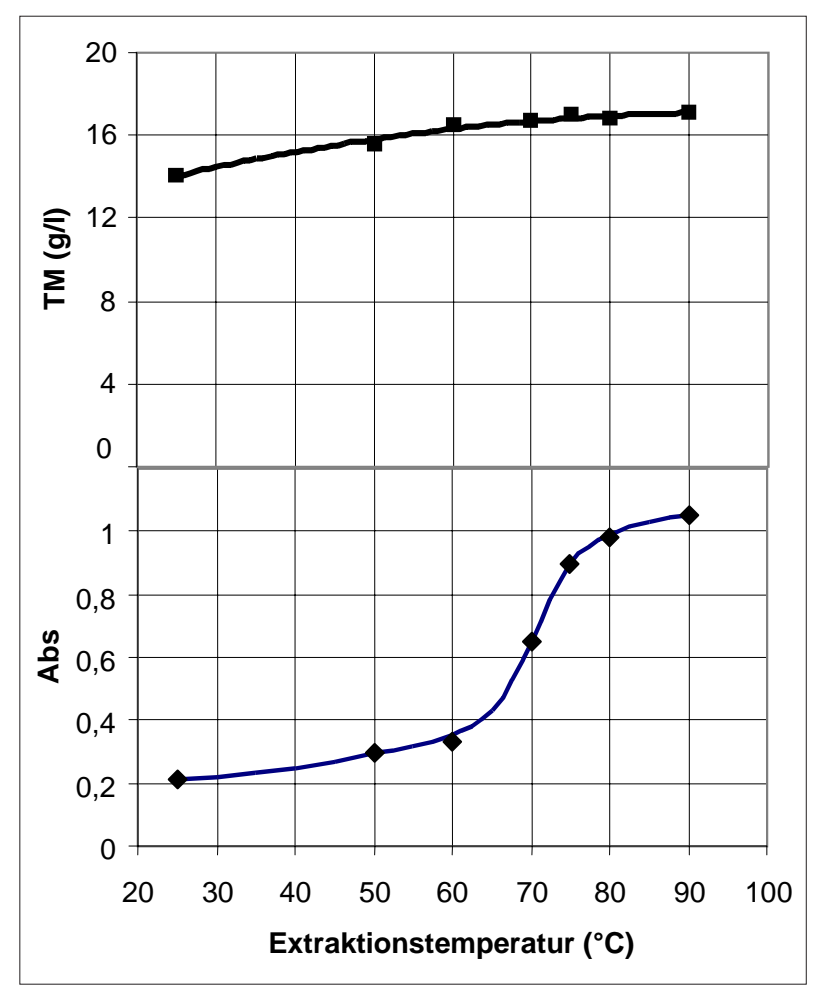

Abb. 7: Extrahierte Trockenmasse (oben) und Absorption bei to (unten) in Abhängigkeit von der Extraktionstemperatur.

Abbildung 8 zeigt den Verlauf der normierten Absorption Abs/Abs $\infty$ bei konstanter Wellenlänge in Abhängigkeit von der Extraktionszeit bei den ersten drei Extraktionsstufen für je ein Beispiel aus den Gruppen hoher und niedriger Extraktionstemperaturen entsprechend Bild 6. Erwartungsgemäß sollte die Extraktion bei hohen Temperaturen schneller ablaufen. Der beispielhafte Vergleich der ersten Stufen ergibt jedoch, dass bei Temperaturen zwischen $25^{\circ} \mathrm{C}$ und $60^{\circ} \mathrm{C}$ der Endwert schneller erreicht wird als bei Temperaturen zwischen $70{ }^{\circ} \mathrm{C}$ und $90{ }^{\circ} \mathrm{C}$. Hingegen bestätigt sich die Erwartung beim Vergleich zweier Isothermen jeweils der zweiten und dritten Stufe.

Bei niedrigen Temperaturen wird das Absorptionsverhalten der ersten Extraktionsstufe von den leicht löslichen Begleitstoffen bestimmt. Der Endwert wird schneller erreicht, da die schwer löslichen Komponenten in der ersten Extraktstufe einen sehr geringen Anteil am Gesamtextrakt ausmachen. Erst mit fortschreitender Abreicherung der leicht löslichen Komponenten in der zweiten und dritten Extraktionsstufe dominiert die Kinetik der schwerer löslichen Farbstoffe das Gesamtverhalten. Die Löslichkeit von Alizarin bei Temperaturen um $50{ }^{\circ} \mathrm{C}$ ist jedoch offenbar so niedrig, dass die Absorption selbst nach einer Extraktionsdauer von ca. $12 \mathrm{~h}$ in der 3. Stufe noch nicht ihren Maximalwert erreicht hat (Abbildung 8 unten).

\section{Färbeergebnisse}

Abbildung 9 zeigt die Ergebnisse von Probefärbungen auf alaungebeizter Baumwolle mit Extrakten unterschiedlicher Extraktionstemperaturen. Für die Darstellung wurden die Werte der Helligkeit L* und des GrünRot-Anteils a* aus dem L*a*b*-Farbsystem ausgewählt.

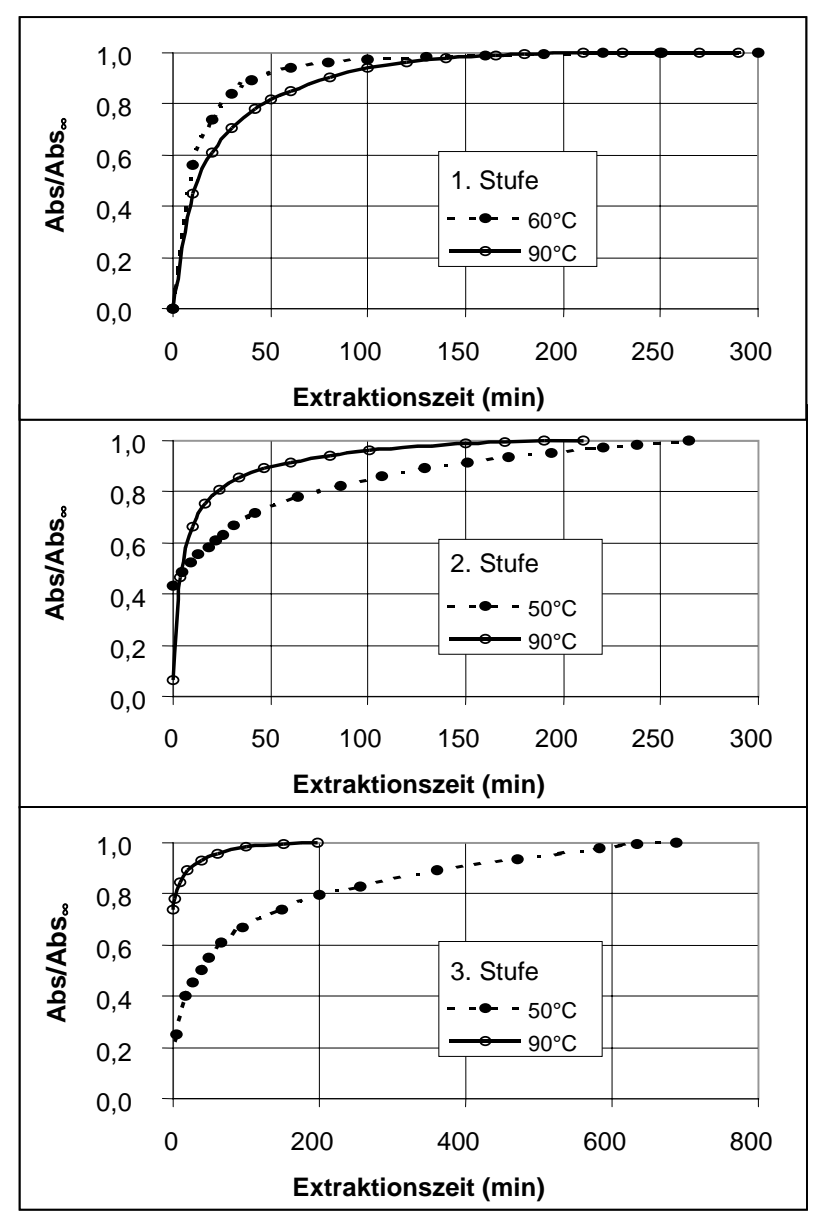

Abb. 8: Normierte Absorption Abs/Abs $\infty$ für je zwei Isothermen der ersten drei Extraktionsstufen in Abhängigkeit von der Extraktionszeit.

Analog zu den Ergebnissen der UV-Vis-Messungen unterscheiden sich Extrakte, die bei $25-60{ }^{\circ} \mathrm{C}$ hergestellt wurden, auch bei Probefärbungen nur wenig. Bei $70{ }^{\circ} \mathrm{C}$ ist aber auch hier ein sprunghafter Anstieg zu verzeichnen, d. h. die Helligkeit der Färbungen sinkt und der RotTon wird intensiver. Oberhalb von $80{ }^{\circ} \mathrm{C}$ ist eine $\mathrm{Ab}$ schwächung der Färbungen zu verzeichnen, obwohl die UV-Vis-Messungen bei $90{ }^{\circ} \mathrm{C}$ die höchsten Farbstoffausbeuten nachwiesen. Hierin zeigt sich die Temperaturempfindlichkeit der Farbstoffextrakte, die bei Temperaturen oberhalb $80{ }^{\circ} \mathrm{C}$ einen merklichen Abbau der Farbstoffe verursacht.

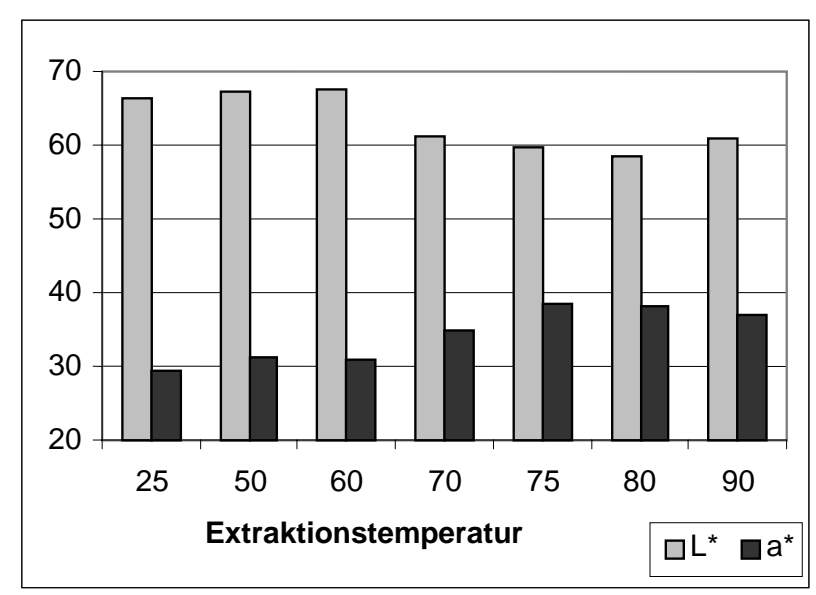

Abb. 9: Farbwerte $L^{*}$ und $a^{*}$ von Probefärbungen mit Extrakten aus der ersten Extraktionsstufe in Abhängigkeit von der Extraktionstemperatur. 
Abbildung 10 stellt die Farbwerte von Probefärbungen mit Extrakten aus drei Extraktionsstufen gegenuiber. Obwohl gemäß Abbildung 5 im Extrakt der zweiten Stufe nur noch halb soviel Farbstoff wie in der ersten Stufe enthalten ist, erweist sich die Färbung mit der zweiten Stufe kaum verändert. Während die Helligkeit leicht ansteigt, bleibt der Rotton nahezu gleich. Wie Abbildung 4 zeigte, erfolgt eine starke Überlagerung der Farbstoffabsorption im Spektrum der 1. Stufe durch nicht färbende Begleitstoffe. Abbildung 7 bestätigt quantitativ das Vorhandensein koextrahierter Begleitstoffe überwiegend in der 1 . Stufe. Dies erklärt auch die qualitativ schlechteren Färbergebnisse mit Extrakten dieser Stufe.

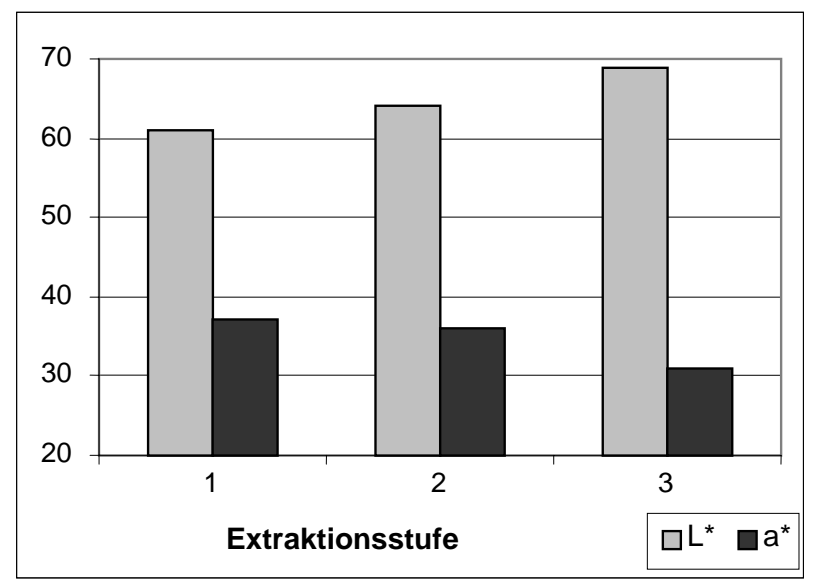

Abb. 10: Farbwerte $L^{*}$ und $a^{*}$ von Probefärbungen mit Extrakten aus den ersten drei Extraktionsstufen bei $T_{\text {extr }}=90^{\circ} \mathrm{C}$.

\section{Zusammenfassung}

In einer Technikumsapparatur mit 15-l-Extraktor und UVVis-Online-Messtechnik wurde der Einfluss der Stufenzahl und der Temperatur auf die Extraktionsausbeute, -kinetik und Färbequalität bei der wässrigen Extraktion von Krappwurzel-Farbstoff untersucht. Es zeigte sich, dass bei $90{ }^{\circ} \mathrm{C}$ mit acht Extraktionsstufen $98 \%$ der Gesamttrockenmasse, aber nur $85 \%$ der eigentlichen Farbstoffe extrahiert werden. Dabei werden ca. 2/3 der Begleitstoffe bereits in der 1 . Stufe extrahiert, eine Extraktionsausbeute, die für den Farbstoffanteil erst nach der 4. Stufe erreicht wird. Wird eine quantitative Kreuzstromextraktion von Krapp angestrebt, so ist eine relativ hohe Zahl von Extraktionsstufen notwendig.

Während der größte Teil der wasserlöslichen Begleitstoffe schon bei niedrigen Temperaturen extrahiert wird, erfordert die quantitative Gewinnung von Farbstoff eine Mindesttemperatur von $75^{\circ} \mathrm{C}$. Die Extraktionsgeschwindigkeit steigt erwartungsgemäß mit der Temperatur, was aber eindeutig nur bei der zweiten und dritten Stufe beobachtet werden konnte. Bei der ersten Stufe wird dieser Trend wegen der massiv koextrahierten, gut löslichen Begleitstoffe umgekehrt.

Die besten Färbeergebnisse wurden mit Extrakten erzielt, die bei $80{ }^{\circ} \mathrm{C}$ gewonnen wurden. Mit dem erststufigen Krapp-Extrakt ließ sich wegen des hohen Anteils an Begleitstoffen keine dem Farbstoffgehalt entsprechend bessere Färbung als in der Folgestufe erzielen.

Zur Verringerung der Stufenzahl bietet sich ein intensiverer Zellaufschluss (mechanisch, fermentativ, Quellung) oder die Verwendung anderer Lösungsmittel an. Für eine Verbesserung der Färbeeigenschaften sollte der Rohstoff so vorbehandelt werden, dass ein günstigeres Verhältnis Farbstoffe/Begleitstoffe entsteht, z. B. mit einer kalten Vorextraktion oder einer fermentativen bzw. enzymatischen Umwandlung der Begleitstoffe.

\section{Danksagung}

Für die finanzielle Förderung dieses Vorhabens sind wir dem Bundesministerium für Bildung, Forschung und Technologie zu Dank verpflichtet.

\section{Literatur}

[1] Abramowski, S.: Extraktion und Trocknung von Inhaltsstoffen der Krapp-Wurzel. Diplomarbeit TFH Wildau, 1998.

[2] Meyer, E.; Abramowski, S.; Schmadl, J.: Extraktion und Trocknung von Krappwurzel-Farbstoff. Wissenschaftliche Beiträge der TFH Wildau, Heft 2/1998

[3] Schweppe, H.: Handbuch der Naturfarbstoffe. ecomed, Landsberg/Lech, 1992

\section{Autoren}

\section{Dr.-Ing. Eckhard Meyer}

Institut fuir Umweltforschung Schlieben

Gartenstr. 43

04936 Schlieben

Tel. (03 53 61) 534

E-Mail: ifu@ifu-schlieben.de

\section{Dipl.-Ing. Jens Uwe Gerking}

Cand. Ing. Torsten Herfort

Prof Dr.-Ing. Josef Schmadl

Technische Fachhochschule Wildau

Technikum für Thermische Verfahrenstechnik

Tel. (0 33 75) 508-110

E-Mail: jschmadl@igw.tfh-wildau.de 